\title{
Maintenance of Endothelial Cell Function in Liquid Based Antithrombotic Surface Coating
}

Hunghao Chu ${ }^{1}$, Jiaqi Yao ${ }^{1}$, Tuo Zhang ${ }^{2}$, Mighten C Yip ${ }^{1}$, Mousumi Dhara ${ }^{1}$, James K Min ${ }^{1}$, Simon Dunham ${ }^{1}$ and Bobak Mosadegh $^{1^{*}}$

${ }^{1}$ Department of Radiology, Dalio Institute of Cardiovascular Imaging, Weill Cornell Medicine \& New York Presbyterian Hospital, 413 E69th St. Suite 108, New York, 10021, USA

${ }^{2}$ Genomics Resources Core Facility, Weill Cornell Medicine, 300 York Ave, New York, USA

"Corresponding author: Bobak Mosadegh, Assistant Professor of Biomedical Engineering in Radiology, Dalio Institute of Cardiovascular Imaging, Weill Cornell Medicine \& New York Presbyterian Hospital, 413 E69th St. Suite 108, New York, 10021, USA, Tel: (949) 202-9981; E-mail: bom2008@med.cornell.edu

Received date: September 27, 2017; Accepted date: October 27, 2017; Published date: November 03, 2017

Copyright: ( $2017 \mathrm{Chu} \mathrm{H}$, et al. This is an open-access article distributed under the terms of the Creative Commons Attribution License, which permits unrestricted use, distribution and reproduction in any medium, provided the original author and source are credited.

\begin{abstract}
Preventing occurrence of thrombosis and biofouling is essential for safety and efficacy of an intravascular device. The slippery liquid-infused porous surface (SLIPS) technology has promise to achieve this goal by forming a liquidrepellent layer on the surface of blood contacting materials. As adhesion of biomolecules is greatly inhibited, the SLIPS-treated surface reduces thrombosing and biofouling. This study demonstrates that in static conditions, cells can adhere to SLIPS-treated surfaces without significant decreases in attachment or migration, as compared to untreated polymer surfaces. Furthermore, next generation RNA sequencing reveals that the SLIPS treatment does not change gene expression in endothelial cells (ECs). Taken together, our findings suggest that SLIPS treatment to be a biocompatible strategy that could potentially protect a variety of medical devices without compromising the process of endothelialization.
\end{abstract}

Keywords: Medical devices; Surface coating; Biocompatibility; Antithrombotic; Endothelial cells

\section{Introduction}

Cardiovascular disease (CVD), known for high morbidity and mortality, represents an immense economic burden on healthcare systems worldwide. In addition to medication, various implantable devices including stents, valves, pacemakers, left ventricular assist devices and vascular grafts are considered to treat most advanced conditions. These devices are highly effective and applied frequently in patients with CVD. However, the host responses following deployment could induce complications like thrombosis and restenosis [1,2]. To minimize these responses, a protective surface coating is crucial for the safety as well as the lifetime of an implanted device. In the case of an intravascular device, an antithrombotic coating is essential to prevent clot deposition during blood circulation [3]. Also, an antifouling coating to avoid infections and inflammatory responses, such as a foreign body reaction, is necessary to maintain function of implanted devices [4].

Slippery liquid-infused porous surface (SLIPS) is a coating technology recently developed to recapitulate liquid-based repellency of pitcher plants [5,6]. Essentially this technology forms a stabilized liquid lubricating layer above a surface and greatly inhibits adhesion of substances to that surface. Three approaches have been developed to stabilize the liquid lubricating layer: i) Applying the liquid to a substrate with micro- or nano-structured features [5]. Once a perfluorocarbon liquid is added, the wicking process can lock the liquid on the surface and form a lubricating layer. ii) Stabilizing the liquid layer using covalently bonded molecules of similar chemistry to the surface. This approach allows pre-existing materials (i.e., medical tubing or devices) to be treated easily by the SLIPS technology, which was achieved by modifying the surface with a perfluorocarbon compound (e.g. tridecafluoro-1,1,2,2-tetrahydrooctyl trichlorosilane) to immobilize the perfluorocarbon liquid (e.g. perfluorodecalin) [7]. iii) Infusing oil into a chemically matched bulk polymer [8-10]. This approach uses the absorption of the oil into the bulk polymer to serve as a reservoir to continually replenish the liquid lubricating layer. Recently, this approach was used to show that cell-sheets can be made by the addition of the oil after a monolayer is formed on the bulk polymer [11]. The SLIPS-treated surface holds additional advantages including high stability and optical transparency as well as being simple, efficient and cost effective to fabricate. Two medical applications derived from liquid repellency of the SLIPS technology include: i) Antifouling: the SLIPS treatment has revealed a superior effect in preventing absorption of proteins and microorganisms than PEGylation, which is a standard antifouling procedure [12,13]; ii) Antithrombotics: the SLIPS treatment has been applied in a variety of medical-grade materials such as polycarbonate and polyvinyl chloride and greatly decreases thrombogenicity of the individual substrates [7].

While this technology is highly effective in reducing biofouling and thrombogenicity, its effects do not last indefinitely when under flow, as would be in the case of blood-contacting medical devices [14]. There still is a need for the body's natural protective surface coating, endothelial cells (ECs), to integrate with this technology to provide long-lasting anti-thrombotic effects. Here, we show for the first time that cells can be seeded onto a SLIPS coated surface, and still be able to attach, grow, and migrate. Here, cell-based assays as well as next generation sequencing were conducted to investigate in vitro biocompatibility of the SLIPS-treated surface. We focused on the compatibility of seeding ECs using the second approach for making SLIPS because a research direction of this technology lies in intravascular devices [15]. 


\section{Materials and Methods}

\section{Materials}

The PDMS layer was made by Sylgard 184 Silicone Elastomer Kit (Dow Corning, Midland, MI). The SLIPS-treated surface was prepared following the published method [7]. Briefly, PDMS or the glass coverslip (Chemglass Life Sciences, Vineland, NJ) was exposed to oxygen plasma and then immersed in anhydrous ethanol (SigmaAldrich, St. Louis, MO) containing 5\% (v/v) tridecafluoro-1,1,2,2tetrahydrooctyl trichlorosilane (Sigma-Aldrich, St. Louis, MO) for 1 hour. The silanized surface was washed with anhydrous ethanol, nanopure water and pure ethanol (Decon Laboratories, King of Prussia, PA). After the residual solvent was removed by the compressed air, the samples were dried at $60^{\circ} \mathrm{C}$ for overnight. To form the lubricating layer, perfluorodecalin (FluoroMed, Round Rock, TX) was added to the silanized surface, followed by three washing steps using DPBS (Thermo Fisher Scientific, Waltham, MA). To coat PDMS or the glass coverslip with gelatin, plasma-exposed PDMS or the coverslip was immersed in DPBS containing $0.2 \%(\mathrm{v} / \mathrm{v})$ gelatin (Thermo Fisher Scientific, Waltham, MA) for 2 hours. After washing the substrates three times with DPBS, the samples were used directly.

\section{In vitro biocompatibility}

The human umbilical vein cell line, EA.hy926 (ATCC, Manassas, Virginia), was maintained in DMEM (Thermo Fisher Scientific, Waltham, MA) containing 10\% FBS (Corning, Corning, NY) and $1 \%$ penicillin/streptomycin (Corning, Corning, NY). For the MTS assay, $5.0 \times 10^{3}$ or $1.0 \times 10^{4}$ cells were seeded in individual surfaces (TCPS, plasma-exposed PDMS, gelatin-coated PDMS and SLIPS-treated) and incubated for 48 hours. After washing once with DPBS, the MTS reagent (Promega, Madison, WI) was added for 2 hours and the absorbance at $490 \mathrm{~nm}$ was recorded by SpectraMax M2 (Molecular Devices, Sunnyvale, CA). To quantify the cell number, ECs were fixed in pure ethanol at $-20^{\circ} \mathrm{C}$ for overnight. After washed three times with DPBS, ECs were stained by $1 \mu \mathrm{g} / \mathrm{ml}$ of PI solution (Biotium, Fremont, CA) for 15 minutes. The fluorescent images were taken by LSM 700 (Zeiss, Oberkochen, Germany) and the intensities (ex/em: $530 \mathrm{~nm} / 620$ $\mathrm{nm}$ ) were recorded by the plate reader. For live cell staining, $2.5 \mu \mathrm{M}$ of calcein AM (Biotium, Fremont, CA) was added in each well and incubated for 30 minutes before the fluorescent intensities (ex/em: 495 $\mathrm{nm} / 517 \mathrm{~nm}$ ) were recorded by the plate reader.

\section{Morphology}

Glass coverslips with three individual surfaces (plasma-exposed, gelatin-coated and SLIPS-treated) were prepared and kept in a 24-well plate. $2.5 \times 10^{4}$ or $5.0 \times 10^{4} \mathrm{ECs}$ were then seeded and incubated for 48 hours. For actin staining, ECs were fixed in PBS containing $4 \%$ paraformaldehyde (EMD Millipore, Hayward, CA) and permeabilized with $0.1 \%$ Triton X-100 solution (Sigma-Aldrich, St. Louis, MO). The DPBS solution containing Rhodamine phalloidin (Thermo Fisher Scientific, Waltham, MA) was added for 15 minutes.

\section{EC migration}

For the EC migration assay, a $5 \mathrm{~mm}$ biopsy punch (Integra Miltex, York, PA) was utilized to create two regions (inside and outside) in a plasma-exposed PDMS stamp. The SLIPS treatment was performed inside the biopsy while the outside was left untreated. ECs were seeded on the untreated PDMS surface and maintained in the culture medium. Once ECs became confluent, the biopsy was removed to allow migration from the untreated to the SLIPS-treated surface. Bright-field and fluorescent images were taken after 7 days.

\section{RNA extraction and Next generation sequencing}

ECs were cultured on the untreated or SLIPS-treated surface for 10 days. Once the cells were harvested, total RNAs were extracted using RNeasy Kit (Qiagen, Hilden, Germany). The concentration and quality of the extracted RNAs were measured by RNA Nano Bioanalyzer (Agilent Technologies, Santa Clara, CA). cDNA libraries were generated using TruSeq RNA Sample Preparation kit v2 (Illumina, San Diego, CA), and were then sequenced using single-end 50bp reads on HiSeq4000 (Illumina, San Diego, CA). The reads were aligned to the UCSC hg19 reference genome using Tophat2 [16]. Gene expressions were measured in RPKM using cufflinks [17].

\section{Results}

Firstly, we investigated the attachment and viability of ECs in comparison to various surfaces. We cultured human ECs on four different surfaces, tissue culture polystyrene (TCPS), untreated polydimethylsiloxane (PDMS), gelatin-coated PDMS and SLIPStreated PDMS, and conducted an MTS assays at 48 hours. TCPS is used as the gold standard for growth of these cells; however, PDMS is a more representative material for comparison, since it is frequently used in microfluidic cell culture systems and has similarities with many polymer-based medical materials [18]. Our result indicated that ECs on SLIPS-treated PDMS revealed similar attachment with those on untreated PDMS and gelatin-treated PDMS at both the high and low cell densities (Figure 1A). To confirm the total number of attached cells were equal for these conditions, we fixed the cells and stained them with propidium iodide (PI) (Figure 1B). The measured fluorescent values indicated that the untreated PDMS and SLIPS-treated PDMS groups had no difference in total cell number (Figure 1C). To ensure cell viability was not compromised, we cultured ECs on gelatin-coated and SLIPS-treated glass coverslips. By live cell staining and quantification, we confirmed that the SLIPS treatment did not compromise cell viability as the untreated and SLIPS-treated groups revealed no difference in the fluorescence values by staining with calcein AM (Figure 1D). To ensure that SLIPS was present prior to seeding the cells, we visualized the movement of a droplet of PBS within a 96 well plate and on coated glass slides (Supplementary M1).

Since the SLIPS treatment greatly changed the surface properties of the underlying substrate, we next asked whether the morphology of cell attachment would be affected by this procedure. We then observed cell morphology by actin staining on glass, gelatin and SLIPS-treated substrates. Compared to the untreated and gelatin-coated surfaces, ECs on SLIPS-treated surface revealed similar morphology (Figure $1 \mathrm{E})$. The images of all three groups indicated that ECs could spread and attach well on the surface, and reached confluence.

Once an intravascular device is implanted, coverage of ECs is crucial for preventing the surface from long-term coagulation and thrombosis [19]. This process relies on the host ECs to populate and proliferate on the surface of the device. To study whether the SLIPS treatment compromises cell migration, we applied a migration assay in which a ring of confluent ECs were exposed to the SLIPS-treated surface by removal of a physical barrier (Figure 2A). After a 7 day culture, we observed a large number of cells on the SLIPS-treated surface suggesting the SLIPS treatment did not inhibit cell migration 
Citation: Chu H, Yao J, Zhang T, Yip MC, Dhara M, et al. (2017) Maintenance of Endothelial Cell Function in Liquid Based Antithrombotic

(Figure 2B). A cell tracking fluorescent dye indicated repopulated ECs were highly viable (Figure $2 \mathrm{C}$ ). A similar result was seen when ECs migrated from the untreated surface to a gelatin-coated PDMS surface (Figure 2D).

Finally, we compared ECs on untreated PDMS or SLIPS-treated PDMS by RNA sequencing. The expression profile of each group consists of 19,182 known transcripts. In general, we found the difference between the untreated and SLIPS-treated groups to be very small (Figure 3). We then determined the cutoff value of fold change to be 1.5 and ranked genes that had the values over 1.5 (Table 1). The gene that had the highest fold change (6.62) was $c$-FOS, which was upregulated in ECs on the SLIPS-treated surface. FOSB, another member in $F O S$ gene family, had 2.56 fold changes while other two members, FOSL1 and FOSL2 did not change significantly (1.06 and 1.12 fold change, respectively) [20]. $c$-FOS-encoded protein interacts with the member of JUN family proteins to form AP- 1 , which is a transcription factor participating in many cellular activities including proliferation, differentiation and transformation [21]. To understand possible effects resulting from upregulation of $c$-FOS or $F O S B$, we checked the levels of $J U N$ genes $(J U N, J U N B$ and $J U N D$ ) and found there was no obvious change between the untreated and SLIPS-treated groups (Table 2). In addition, AP-1 regulates several genes that control cell survival and death [22]. We examined the levels of these genes and found change to be small as well. As a result, we considered the possible adverse effects due to upregulation of $c-F O S$ can be avoided.

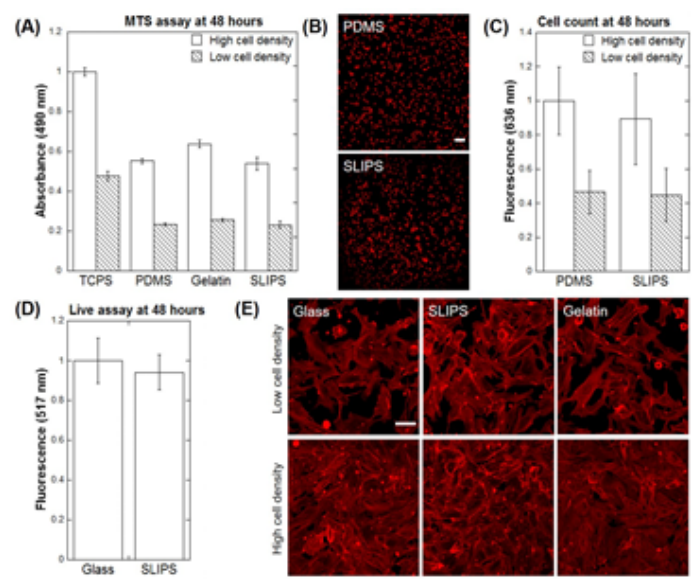

Figure 1: (A) MTS cell proliferation assay. High (open bars) and low (hatched bars) densities of ECs were seeded on TCPS, untreated PDMS, gelatin-coated PDMS and SLIPS-treated PDMS. After $48 \mathrm{~h}$ culture, MTS assays were conducted to compare cell proliferation (mean $\pm \mathrm{SD}, \mathrm{n}=8)$. (B, C) Fluorescent images (10X) of cells stained by PI. Comparison of fluorescent values between the untreated and SLIPS-treated groups found no statistical difference (mean \pm SD, $\mathrm{n}=8$ ). Scale bar: $100 \mu \mathrm{m}$. (D) Live cell staining of ECs on untreated and SLIPS-treated glass surfaces. Comparison of fluorescent values revealed no difference these two groups (mean $\pm S D, n=5$ ). (E) Confocal fluorescence images (20X) of ECs stained with rhodamine-conjugated phalloidin. ECs cultured on three surfaces showed similar morphology at low (top) and high (bottom) cell densities $\left(2.5 \times 10^{4}\right.$ and $5.0 \times 10^{4}$ cells, respectively). Scale bar: 100 $\mu \mathrm{m}$.

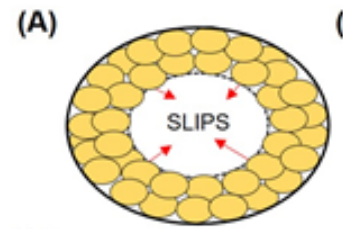

(C)

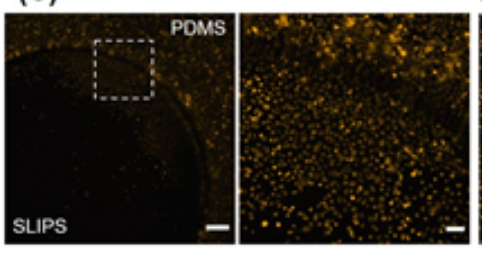

(B)

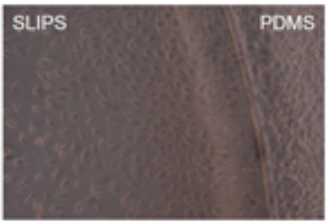

(D)

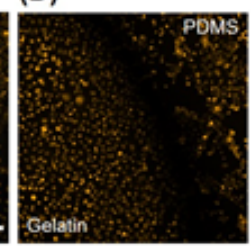

Figure 2: (A) Scheme of a migration assay. The untreated PDMS surface was separated from the SLIPS-treated surface by a biopsy punch. Once ECs (yellow) on the untreated PDMS surface became confluent, the biopsy punch was removed to allow cells to migrate to the SLIPS-treated surface. (B) Light micrograph of ECs migrating from the untreated surface to SLIPS-treated surface. (C) Confocal fluorescence images (left: 2.5X; right: 10X) of ECs stained with CellTracker $^{\text {ma }}$ Orange CMRA. Enzymatic cleavage of CMRA in viable ECs converted it to a fluorescent product, which retained inside the cells. Scale bars: $500 \mu \mathrm{m}(2.5 \mathrm{X}) ; 100 \mu \mathrm{m}$ (10X). (D) Confocal fluorescence image (10X) of ECs migrating from untreated PDMS to gelatin-coated PDMS.

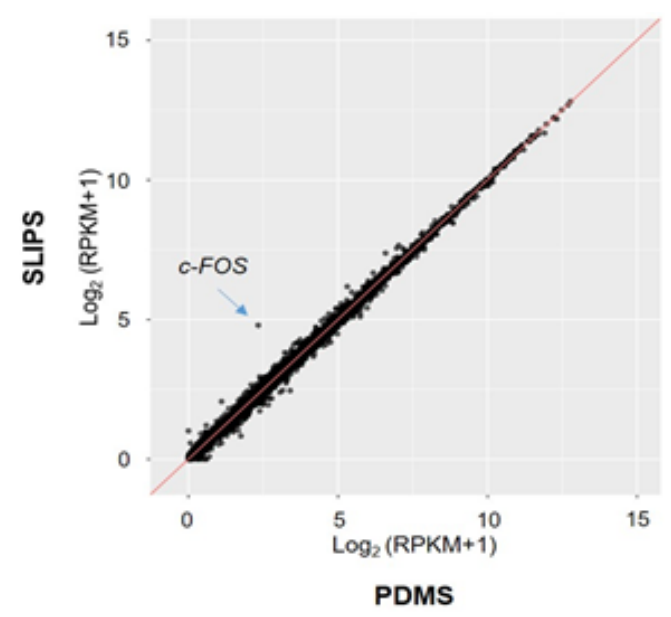

Figure 3: Scatter plot of PDMS Log2 (RPKM+1) versus SLIPS Log2 $(\mathrm{RPKM}+1)$. RPKM: reads per kilobase of transcript per million mapped reads. The dot represented c-FOS whose expression was highly unregulated in the SLIPS-treated group. 
Citation: Chu H, Yao J, Zhang T, Yip MC, Dhara M, et al. (2017) Maintenance of Endothelial Cell Function in Liquid Based Antithrombotic Surface Coating. Biol Med (Aligarh) 9: 418. doi:10.4172/0974-8369.1000418

Page 4 of 7

\begin{tabular}{|c|c|c|c|c|}
\hline & Gene_ID & RPKM (PDMS) & RPKM (SLIPS) & Fold change \\
\hline \multirow{27}{*}{ Upregulation } & FOS & 4.04 & 26.78 & 6.62 \\
\hline & EGR1 & 1.15 & 3.18 & 2.76 \\
\hline & FOSB & 0.51 & 1.31 & 2.56 \\
\hline & GDPD3 & 0.49 & 0.98 & 2.01 \\
\hline & FN1 & 38.72 & 71.46 & 1.85 \\
\hline & LRRC71 & 0.51 & 0.93 & 1.83 \\
\hline & STATH & 0.54 & 0.94 & 1.75 \\
\hline & HSPG2 & 95.39 & 165.39 & 1.73 \\
\hline & GABRE & 0.92 & 1.56 & 1.7 \\
\hline & DHRS2 & 3.28 & 5.33 & 1.63 \\
\hline & PILRB & 1.55 & 2.51 & 1.62 \\
\hline & LRP1 & 4.22 & 6.74 & 1.6 \\
\hline & socs3 & 8.34 & 13.08 & 1.57 \\
\hline & ARHGAP11B & 0.77 & 1.21 & 1.57 \\
\hline & ZFP36 & 10.54 & 16.34 & 1.55 \\
\hline & PTGS1 & 1.19 & 1.84 & 1.55 \\
\hline & CYR61 & 129.83 & 200.58 & 1.54 \\
\hline & TNFRSF25 & 2.65 & 4.08 & 1.54 \\
\hline & CPT1B & 1.07 & 1.64 & 1.53 \\
\hline & AHSA2 & 1.36 & 2.07 & 1.53 \\
\hline & TOX & 2.9 & 4.41 & 1.52 \\
\hline & GABBR1 & 0.69 & 1.05 & 1.52 \\
\hline & ZNF789 & 0.69 & 1.04 & 1.51 \\
\hline & CTGF & 123.98 & 186.92 & 1.51 \\
\hline & LTB4R2 & 0.61 & 0.92 & 1.5 \\
\hline & LCAT & 2.19 & 3.29 & 1.5 \\
\hline & FBN2 & 11.27 & 16.91 & 1.5 \\
\hline \multirow{8}{*}{ Downregulation } & NRSN1 & 2.37 & 0.78 & 3.05 \\
\hline & HIST1H2AG & 1.01 & 0.44 & 2.28 \\
\hline & IL8 & 9.57 & 4.48 & 2.13 \\
\hline & IL32 & 2.17 & 1.04 & 2.08 \\
\hline & HIST1H4H & 1.82 & 0.97 & 1.88 \\
\hline & HLA-DRA & 5.34 & 2.85 & 1.87 \\
\hline & HIST1H2BJ & 1.43 & 0.78 & 1.83 \\
\hline & CD74 & 4.22 & 2.31 & 1.83 \\
\hline
\end{tabular}


Citation: Chu H, Yao J, Zhang T, Yip MC, Dhara M, et al. (2017) Maintenance of Endothelial Cell Function in Liquid Based Antithrombotic Surface Coating. Biol Med (Aligarh) 9: 418. doi:10.4172/0974-8369.1000418

Page 5 of 7

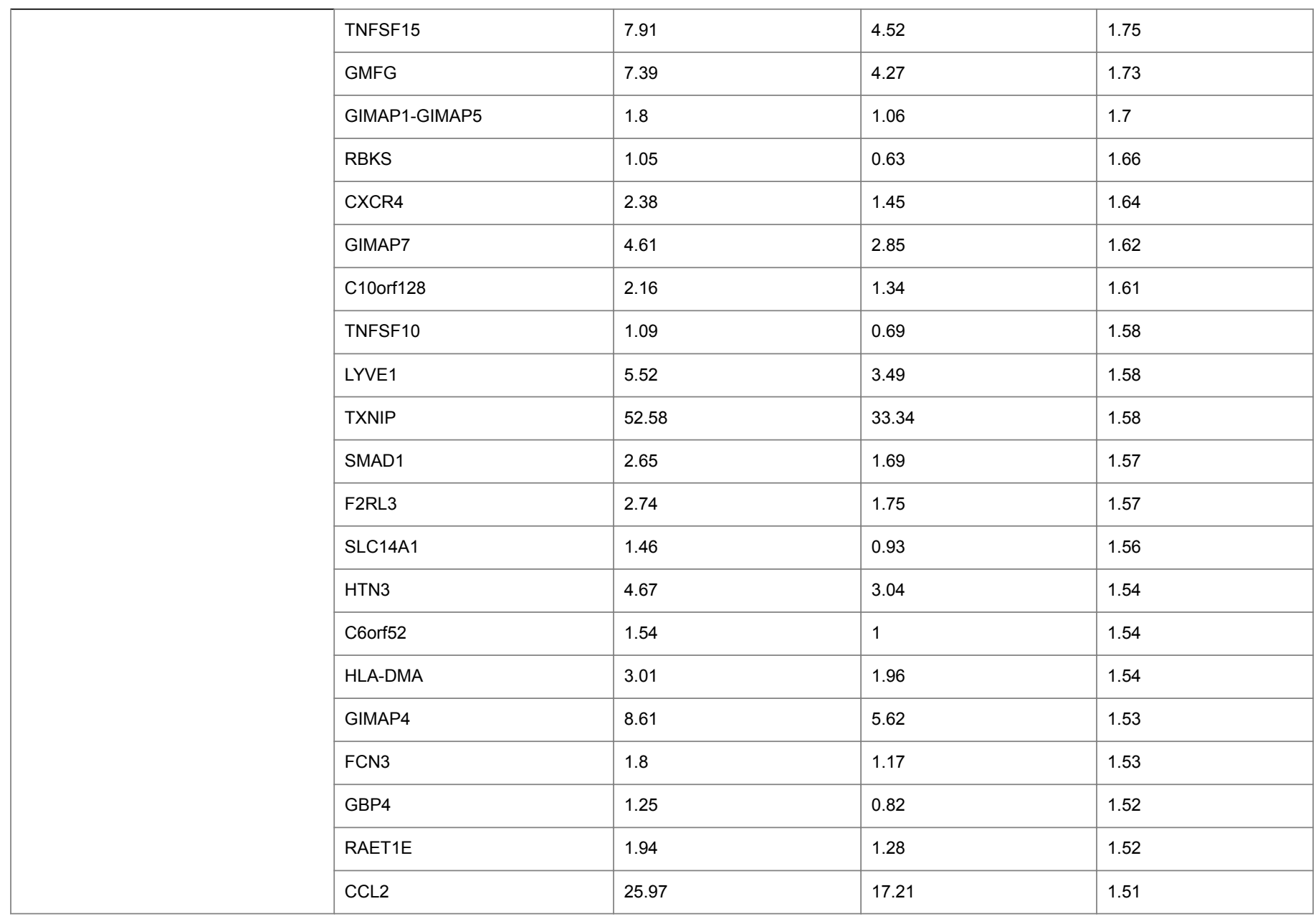

Table 1: Summary of upregulated and downregulated genes that had fold change above 1.5. For RPKM (PDMS) $>$ RPKM (SLIPS), fold change was calculated by RPKM (PDMS)/RPKM (SLIPS). For RPKM (PDMS)<RPKM (SLIPS), fold change was calculated by RPKM (SLIPS)/RPKM (PDMS).

\begin{tabular}{|c|c|c|c|c|}
\hline & Gene_ID & RPKM (PDMS) & RPKM (SLIPS) & Fold change \\
\hline \multirow{3}{*}{ FOS } & JUN & 54.73 & 54.43 & 1.01 \\
\hline & JUNB & 25.95 & 28.97 & 1.12 \\
\hline & JUND & 37.9 & 35.27 & 1.07 \\
\hline \multirow{8}{*}{ AP-1 } & CCND1 & 277.19 & 294.78 & 1.06 \\
\hline & TP53 & 53.42 & 54.16 & 1.01 \\
\hline & CDKN1A & 169.31 & 174.25 & 1.03 \\
\hline & CDKN2A & n.s. & n.s. & \\
\hline & CSF2 & 4.91 & 4.34 & 1.13 \\
\hline & FGF7 & n.s. & n.s. & \\
\hline & HBEGF & 18.36 & 22.9 & 1.25 \\
\hline & FASLG & n.s. & n.s. & \\
\hline
\end{tabular}




\begin{tabular}{|l|l|l|l|l|}
\hline \multirow{2}{*}{} & FAS & 9.36 & 7.79 & 1.2 \\
\cline { 2 - 6 } & BCL3 & 16.71 & 16.44 & 1.02 \\
\hline
\end{tabular}

Table 2: Representative genes participating in FOS signaling. FOS dimerizes with JUN to form AP-1, which regulates a class of genes involved in cell survival and death. For RPKM (PDMS)>RPKM (SLIPS), fold change was calculated by RPKM (PDMS)/RPKM (SLIPS). For RPKM (PDMS) $<$ RPKM (SLIPS), fold change was calculated by RPKM (SLIPS)/RPKM (PDMS). n.s.: not significant due to very low expression $(\mathrm{RPKM}<0.05)$.

\section{Discussion}

The SLIPS technology employs different approaches to immobilize the perfluorocarbon liquid on the substrate, which creates a stable and lubricating surface. Perfluorocarbon compounds have many features that lead to many biomedical applications. For example, they are chemically inert and extremely stable in physiological environments even though their metabolism is not fully understood [23]. In addition, perfluorocarbons have high solubility to many gases including oxygen. Thus, they act as artificial oxygen carriers and are utilized in human ischemic conditions [24]. Also, perfluorocarbons are both hydrophobic and lipophobic, thus, they show high efficacy in protecting a medical device and enhancing its efficiency [25]. It is anticipated that more biomedical applications employing perfluorocarbons will be explored in the coming future. Consequently, investigating their biocompatibility from all aspects is necessary for the adoption of this technology.

Established studies demonstrate antifouling and antithrombotic benefits of the SLIPS-treated surface [7,12,13]. Antifouling and antithrombotic efficacy results from the slippery surface to repel adhesion of biomolecules. It seems to correlate with low cell attachment and thus poor cell proliferation. In contrast, the results of this study suggest that SLIPS-treated PDMS or glass does not compromise cell attachment in comparison with the untreated substrate. These two processes could possibly be explained by different mechanisms. When proteins or microorganisms flow through the SLIPS-treated surface, the lubricating layer separates them from the underlying substrate. Due to their interaction with the lubricating compound being weak, complete removal from the surface is efficient, as demonstrated in previous work [5]. In the case of in vitro cell culture, we expect that gravity pulls the cells into contact with the underlying surface, without significant interference from the lubricating compound when in a static condition. Furthermore, we suspect that the fluorination of the surface is not a perfect monolayer, possibly exposing pockets of attachment sites. Collectively, we propose that the SLIPS treatment provides dual benefits to an intravascular device (Figure 4). (i) The SLIPS-treated surface prevents the formation of thrombus on the device. Its efficacy does not sustain for a long period of time due to consumption of the lubricating compound under flow conditions [14]. (ii) The long-term antithrombotic effect would come from host ECs, which migrate to cover the surface of the device [26]. In the current study, we demonstrate ECs can attach and migrate on the SLIPS-treated surface. Future studies are needed to show how well the ECs proliferate over long periods, can remain attached under flow conditions, and effect the SLIPS layer as they proliferate on the surface. In addition, future studies can investigate the mechanism by which cells are able to attach to SLIPS-coated surfaces, since the exact mechanisms are not understood. The current study uses an endothelial cell line as a proof-of-principle that mammalian cells can attach, with little phenotypic effects, to SLIPS-treated substrates under static conditions, but confirmation with primary cells will ensure translation of this technology. Since EC activity is critical for the long-term efficacy of blood-contacting medical devices, we envision future work in modifying the surface with cell adhesion molecules to promote these processes [27,28].

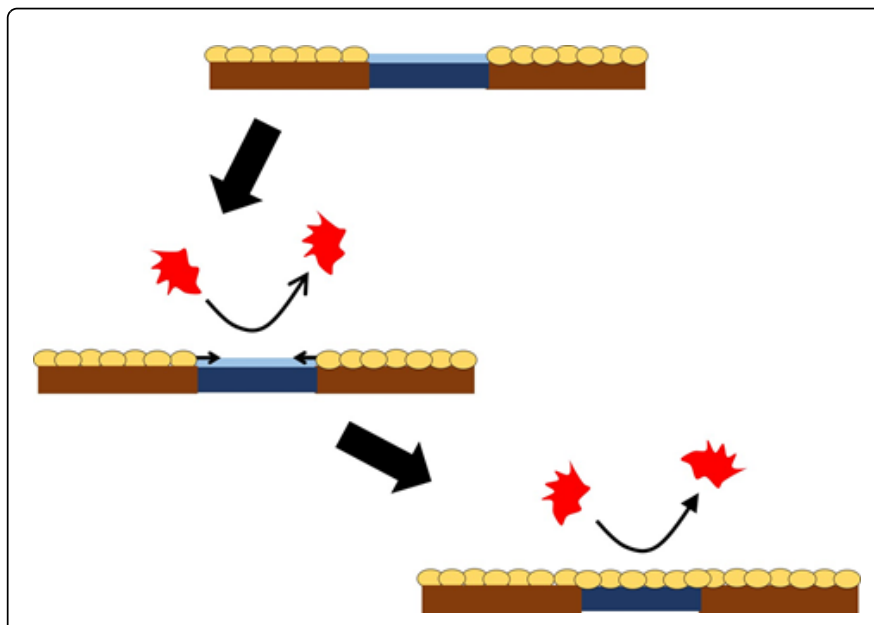

Figure 4: Scheme of the proposed dual-functioned SLIPS-treated surface. Once implanted, the lubricating layer (light blue) prevents the formation of thrombus or biofouling (red) on a medical device (dark blue). As host ECs (yellow) could migrate from the surrounding regions to the SLIPS-treated surface, ultimately an EC monolayer is formed to prevent long-term failure of the device.

\section{Acknowledgements}

We thank the Dalio ICI for funding this work.

\section{Author Contributions Statement}

H.C., J.Y. M.Y., M.D. and T.Z. conducted the experiments; B.M., and H.C. and analyzed the results and co-wrote the manuscript. J.Y. and T.Z. performed RNA experiments; B.M., S.D., and J. M. oversaw project.

\section{References}

1. Anderson JM, Rodriguez A, Chang DT (2008) Foreign body reaction to biomaterials. Semin Immunol 20: 86-100.

2. Bluestein D, Chandran KB, Manning KB (2010) Towards nonthrombogenic performance of blood recirculating devices. Ann Biomed Eng 38: 1236-1256.

3. de Mel A, Cousins BG, Seifalian AM (2012) Surface modification of biomaterials: a quest for blood compatibility. Int J Biomater 2012: 707863. 
Citation: Chu H, Yao J, Zhang T, Yip MC, Dhara M, et al. (2017) Maintenance of Endothelial Cell Function in Liquid Based Antithrombotic Surface Coating. Biol Med (Aligarh) 9: 418. doi:10.4172/0974-8369.1000418

Page 7 of 7

4. Meyers SR, Grinstaff MW (2012) Biocompatible and bioactive surface modifications for prolonged in vivo efficacy. Chem Rev 112: 1615-1632.

5. Wong TS, Kang SH, Tang SKY, Smythe EJ, Hatton BD, et al. (2011) Bioinspired self-repairing slippery surfaces with pressure-stable omniphobicity. Nature 477: 443-447.

6. Sotiri I, Overton JC, Waterhouse A, Howell C (2016) Immobilized liquid layers: A new approach to anti-adhesion surfaces for medical applications. Exp Biol Med 241: 909-918.

7. Leslie DC, Waterhouse A, Berthet JB, Valentin TM, Watters AL, et al. (2014) A bioinspired omniphobic surface coating on medical devices prevents thrombosis and biofouling. Nat Biotech 32: 1134-1140.

8. Yao X, Dunn SS, Kim P, Duffy M, Alvarenga J, et al. (2014) Fluorogel elastomers with tunable transparency, elasticity, shape-memory, and antifouling properties. Angew Chem Int Ed 53: 4418-4422.

9. Howell C, Vu TL, Lin JJ, Kolle S, Juthani N, et al. (2014) Self-replenishing vascularized fouling-release surfaces. ACS Appl Mater Interfaces 6: 13299-13307.

10. MacCallum N, Howell C, Kim P, Sun D, Friedlander R, et al. (2015) Liquid-infused silicone as a biofouling-free medical material. ACS Biomater Sci Eng 1: 43-51.

11. Juthani N, Howell C, Ledoux H, Sotiri I, Kelso S, et al. (2016) Infused polymers for cell sheet release. Sci Rep 6: 26109.

12. Epstein AK, Wong TS, Belisle RA, Boggs EM, Aizenberg J (2012) Liquidinfused structured surfaces with exceptional anti-biofouling performance. Proc Natl Acad Sci USA 109: 13182-13187.

13. Chen J, Howell C, Haller CA, Patel MS, Ayala P, et al. (2017) An immobilized liquid interface prevents device associated bacterial infection in vivo. Biomaterials 113: 80-92.

14. Howell C, Vu TL, Johnson CP, Hou X, Ahanotu O, et al. (2015) Stability of surface-immobilized lubricant interfaces under flow. Chem Mater 27: 1792-1800.

15. Kono K, Hiruma H, Kobayashi S, Sato Y, Tanaka M, et al. (2016) In vitro endothelialization test of biomaterials using immortalized endothelial cells. PLoS ONE 11: e0158289.

16. Kim D, Pertea G, Trapnell C, Pimentel H, Kelley R, et al. (2013) TopHat2 accurate alignment of transcriptomes in the presence of insertions, deletions and gene fusions. Genome Biol 14: R36-R36.
17. Trapnell C, Williams BA, Pertea G, Mortazavi A, Kwan G, et al. (2010) Transcript assembly and abundance estimation from RNA-Seq reveals thousands of new transcripts and switching among isoforms. Nat Biotechnol 28: 511-515.

18. Teo AJT, Mishra A, Park I, Kim YJ, Park WT, et al. (2016) Polymeric biomaterials for medical implants and devices. ACS Biomater Sci Eng 2: 454-472.

19. Jaffer IH, Fredenburgh JC, Hirsh J, Weitz JI (2015) Medical deviceinduced thrombosis: what causes it and how can we prevent it? J Thromb Haemost 13: S72-S81.

20. Renaud SJ, Kubota K, Rumi MAK, Soares MJ (2014) The FOS transcription factor family differentially controls trophoblast migration and invasion. J Biol Chem 289: 5025-5039.

21. Janknecht R, Cahill MA, Nordheim A (1995) Signal integration at the cfos promoter. Carcinogenesis 16: 443-450.

22. Shaulian E, Karin M (2002) AP-1 as a regulator of cell life and death. Nat Cell Biol 4: E131-E136.

23. Grapentin C, Barnert S, Schubert R (2015) Monitoring the stability of perfluorocarbon nanoemulsions by cryo-TEM image analysis and dynamic light scattering. PLoS ONE 10: e0130674.

24. Spahn DR (1999) Blood substitutes artificial oxygen carriers: perfluorocarbon emulsions. Crit Care 3: R93-R97.

25. Lee YAL, Zhang S, Lin J, Langer R, Traverso G (2016) A janus mucoadhesive and omniphobic device for gastrointestinal retention. Adv Healthcare Mater 5: 1141-1146.

26. Li S, Henry JJD (2011) Nonthrombogenic approaches to cardiovascular bioengineering. Annu Rev Biomed Eng 13: 451-475.

27. Robinet A, Fahem A, Cauchard JH, Huet E, Vincent L, et al. (2005) Elastin-derived peptides enhance angiogenesis by promoting endothelial cell migration and tubulogenesis through upregulation of MT1-MMP. J Cell Sci 118: 343-356.

28. McNichols C, Wilkins J, Kubota A, Shiu YT, Aouadi SM, et al. (2014) Investigating surface topology and cyclic-RGD peptide functionalization on vascular endothelialization. J Biomed Mater Res Part A 102A: 532-539. 